\title{
Neue Horizonte feministischer Wissensproduktion
}

In feministischen Diskussionen wird seit den Anfängen der Zweiten Frauenbewegung Ende der 1960er Jahre das Verhältnis von wissenschaftlicher und bewegungsbezogener Wissensproduktion als ein Verhältnis von ,Theorie und Praxis' diskutiert.[1] Dabei geht es idealerweise auch um eine Anerkennung der unterschiedlichen ,Qualitäten' der verschiedenartigen Wissensformen und -praktiken (handwerkliche, soziale, theoretische, erfahrungsbezogene, künstlerische), verbunden mit dem Versuch, deren Bewertung neu auszutarieren und deren Hierarchisierung herauszufordern. Dies impliziert, die Unterschiedlichkeit sozialer Positionierungen in ihrem sozialen, politischen und ökonomischen Kontext zu reflektieren. Feministische Wissensproduktion kann also bedeuten, Wissensformen in Zusammenhang $\mathrm{zu}$ bringen mit den damit verbundenen ungleichen und hierarchisierten Sprechpositionen und Hegemonien, beispielsweise mit Klassenunterschieden, Rassismus und Heteronormativität. Um diesen hohen Anspruch an die eigene Reflexivität wird in feministischen Debatten immer wieder neu gekämpft. Mein Text beschäftigt sich mit der Entwicklung dieses Anspruchs und skizziert seine Fallstricke. Ich plädiere hier für eine erneute, stärkere Kopplung feministischer Theorie und Praxis, um akademische feministische Wissensproduktion wieder deutlicher der Bewegungspraxis anzunähern und ihre gesellschaftspolitische Relevanz zu erhöhen. Dies macht eine fortgesetzte Auseinandersetzung mit den Mechanismen der Wissensproduktion und der Hierarchisierung feministischer Wissensformen notwendig.

\section{Aus der Theorie in die Praxis: Feministische Wissensproduktion dezentrieren}

Braucht es heute ein Studium der Gender Studies, um sich feministisch zu engagieren? Diese provozierende Frage weist auf die heute stark akademisierte Rezeption feministischen Wissens hin. Zugleich macht sie aber auch deutlich, dass an Hochschulen nicht nur akademisches Wissen weiterentwickelt wird. Diese sind heute nach wie vor Orte, an denen sich feministisch Engagierte in Gruppen und Kontexten versammeln. So sind (nicht nur, aber auch) die 
organisierten Strukturen im AStA (FrauenLesbenTrans*Q Queer-Referate, Feministische Archive, Schwulenreferate) innerhalb der Hochschulen ein wichtiger Rahmen zur Politisierung und für kollektive, politisch-private Aktivitäten, die zum Beispiel in Kooperation mit (sub-)kulturellen, politischen oder gewerkschaftlichen Akteur_innen organisiert werden, wie Veranstaltungen (z. B. Vorträge und Vorlesungsreihen) und das Sammeln praktischer Erfahrungen (z. B. Webdesign-Kurse, Selbstverteidigung).

Universitäten sind allerdings auch heute nicht die einzigen Orte feministischer Wissensproduktion und akademisch Gebildete sind nicht deren einzige Produzent_innen, obwohl sie nicht selten den Alleinvertretungsanspruch darauf erheben und längst eine akademische Hegemonie innerhalb feministischer Wissensformen entstanden ist. Akademisches feministisches Wissen sollte daher wieder stärker dezentriert als eine von vielen feministischen Wissensformen betrachtet werden. Zahlreiche aktuelle queer/feministische Projekte verdeutlichen, dass Feminismus nach wie vor jenseits akademischer Formierung und fern von Themen, die in akademischen Kreisen diskutiert werden, außerordentlich lebendig ist. Feministische Wissensproduktion in der Praxis findet zum Beispiel bei Ladyfesten und Riot-Grrrl-Aktivitäten statt. Sie wird sichtbar in feministischen Internet- und Zeitschriftenprojekten, Aktionen von FEMEN und Protesten von Sexarbeiter_innen, in feministischen Handwerker_innen-Kollektiven, Archiven und den noch existierenden feministischen Buchläden, in Beratungsstellen, in Transgender-Vereinen, Selbstverteidigungsprojekten und Frauenhäusern, in Queer-of-Color-Initiativen, queer/feministischen Bands, Performancegruppen und Chören, in Film- und Radioprojekten sowie feministischen Schreibwerkstätten und Gesundheitszentren, um nur einige Bereiche zu nennen. Der Fokus der Aktivitäten liegt in vielen der genannten Projekte und Initiativen eher auf Praxisnähe und Alltagserfahrung, direktem Austausch und konkreter Unterstützung, auf Information, Aufklärung, politischem Aktivismus und kollektiver Organisierung, aber auch in handwerklichen, gesundheitsbezogenen, kulturellen und künstlerischen Bereichen. Wie schon zu Beginn feministischer politischer Organisierung dienen diese kollektiven Projekte dazu, individuelle Erfahrungen auszutauschen und dadurch zu überschreiten, dass überindividuelle Gemeinsamkeiten entdeckt und in politische Praxis übersetzt werden.

Große Teile des akademischen Feminismus haben allerdings durch die über die Jahrzehnte entstandene Kluft zwischen feministischer Praxis und akademisierter Theorie ein Eigenleben entfaltet und kommen weitgehend ohne Praxisbezug aus. Dies geht mit einer Angleichung an die herkömmliche Wissensproduktion einher, bei der Praxis und Erfahrung zum Forschungsgegenstand werden, anstatt als eigenständige Wissensformen ihren eigenen Beitrag zur feministischen Wissensproduktion zu leisten. Diese Form der Entmündigung der Praxis durch die Theorie führt zu einem einseitigen Theorie-Praxis-Wissenstransfer, in dem sich akademisches feministisches Wissen der Praxis als Interpretationsfolie und zur Untermauerung sozialer und politischer Erfahrungen anbietet, ohne dass umgekehrt eine praxisbasierte Theoriebildung fortgeschrieben würde.

Vor dem Hintergrund der existierenden, differenten feministischen Wissensformen gibt es immer wieder Versuche sowohl von der praxisorientierten als auch von der theorieorientierten feministischen Seite aus, sich zu 
verständigen und miteinander auseinanderzusetzen. Dies kann Konflikte mit sich bringen, z. B. im Hinblick auf das Sprachregister, das bei Veranstaltungen benutzt wird (akademisch oder alltagsnah) sowie auf die Denkbezüge, die dabei zugrunde gelegt werden (theoretisch oder erfahrungsbasiert), besonders, wenn Moderation und Vermittlung verschiedener Wissensbestände fehlen. Dazu ein Beispiel: Bei einer „queerfeministischen Konferenz“ in Berlin, die sich explizit sowohl an akademisches wie nichtakademisches Publikum richtete, kam es vor einigen Jahren zum Konflikt darum, wie Wissen kommuniziert und produziert werden sollte. Einige Teilnehmer_innen ohne akademische Vorbildung kritisierten die Veranstaltung als „zu akademisch geprägt“, da bereits Sprachduktus und theoretische Referenzen einiger Referent_innen nur akademisch Gebildeten zugänglich wären, was Nicht-Akademiker_innen aus den Diskursen ausschloss. Sie erlebten dabei ihr erfahrungsbasiertes, praktisches Wissen als dem akademischen Wissen unterlegen und machten die Erfahrung, von akademisch vorgebildeten Teilnehmer_innen untergeordnet zu werden. In einer spontan anberaumten Diskussion wurde diese Diskrepanz und Schräglage noch während der Konferenz diskutiert. Bereits der Versuch und die Offenheit, über entsprechende Wissenshierarchien und deren soziale Konsequenzen zu sprechen, lassen sich aus meiner Sicht als Besonderheit queer/feministischer Kontexte bewerten. Sie verdeutlichen den hohen selbstreflexiven Anspruch, der zumindest in einigen Kreisen an der Schnittstelle von queer/feministischer Theorie und Praxis nach wie vor besteht (vgl. Schuster 2010: 268 ff.).

\section{Feministisches Wissen}

Feministische Wissensproduktion unterscheidet sich aufgrund ihres Anspruchs, sich mit dem Spannungsfeld von Theorie und politischer Praxis auseinanderzusetzen, von vielen anderen wissenschaftlichen Kontexten. Dies hängt mit ihrer Besonderheit zusammen: Sie gründet auf der Praxis politischer und erfahrungsbasierter Theorieentwicklung. Diese Praxis ist nicht eigens durch feministische Akteur_innen entwickelt worden, sondern lässt sich zumindest im US-amerikanischen Kontext auf die politische Praxis Schwarzer Frauen in den Bürgerrechtsbewegungen zurückführen (Phipps 2016: 2 f.). Ähnliche erfahrungsbasierte Wissensproduktion findet sich auch etwa in den Ansätzen der Pädagogik der Unterdrückten von Paulo Freire (1971) und weiteren befreiungspädagogischen Konzepten weltweit. Es lassen sich zudem paradigmatische Parallelen zu Forschungsansätzen etwa in der Aktionsforschung ziehen, die versucht, soziale Bewegungen und Aktivist_innen gleichberechtigt mit den Forschenden an der Erhebung und Produktion von Forschungsdaten zu beteiligen, wobei die Forschung ihrerseits auf gesellschaftliche Veränderungen im Sinne der Beteiligten zielt (Kindon/Pain/Kesby 2009).

Feministische Theorienproduktion ist also aus der Wissensproduktion vielstimmiger politischer Bewegungen hervorgegangen, die seit ihren Anfängen praxisorientiert, interdisziplinär und institutionenkritisch organisiert und ausgerichtet sind. Dies bedeutete immer auch, dass einige Erfahrungen mehr Gewicht und Gehör erhielten als andere, und dadurch mitunter eine Marginalisierung bestimmter Erfahrungen und Positionen zugunsten anderer.[2] In diesem Zusammenhang kritisieren Women of 
Color und Feministinnen des Globalen Südens seit Ende der 1970er Jahre die Essentialisierung ,der ' weiblichen Erfahrung, die eine Marginalisierung Schwarzer bzw. nicht-,westlicher Frauen und damit eine Homogenisierung differenter Erfahrungen und Politiken innerhalb feministischer Bewegungen erzeugt. Diese Interventionen sind frühe Hinweise auf intersektionale Verschränkungen. Sie führten zusammen mit der grundsätzlichen Kritik der Gegenüberstellung von sex als biologisch bedingt und gender als kulturell konstituiert und der Analyse heteronormativer Gesellschaftsstrukturen in poststrukturalistischen feministischen Theorien vonseiten Lesbian/Queer/ Trans*-Feminist_innen seit den 1990er Jahren zur Herausbildung von queer/feministischen und später zu trans/feministischen Theorien, letzteren insbesondere in anglophonen und spanischsprachigen Kontexten (Stryker/ Bettcher 2016). Bis heute ist in feministischen Diskursen umkämpft, wer als Subjekt des Feminismus sprechen und agieren darf. Dabei spielen gesellschaftliche Hierarchien eine bedeutsame Rolle, insbesondere rassistische Ausschlüsse und die Diskriminierung von Trans*-Personen, aber auch Heteronormativität und Klassenfragen.[3]

Irene Dölling (2013) unterscheidet wissenschaftlichen von akademischem Feminismus, wobei sich Letzterer später aus Ersterem entwickelte. Der wissenschaftliche Feminismus entsteht in den USA und in Europa seit den Anfängen der Zweiten Frauenbewegung in den 1960er und 7oer Jahren auf der Basis erfahrungsbasierter Theorieproduktion, als Frauen[4] gemeinsam mit anderen Frauen durch Selbsterfahrung und Erfahrungsaustausch begannen, über die eigene Subjektivität zu reflektieren (Hark 2005: 227). In diesem kollektiven Wissensproduktionsprozess erschienen nun scheinbar private Themen als gemeinsame. Es wurden Rückschlüsse auf strukturelle Bedingungen gezogen („Das Private ist politisch!“) und Themen politischer Kämpfe definiert. Zugänge, die bisher in hegemonialen Diskursen ausgeblendet waren, wurden in den Fokus gestellt. Ein zentrales Feld bildete dabei die Kritik an der kapitalistisch organisierten und geschlechtsspezifischen Verteilung von Erwerbs- und Reproduktionsarbeit und ihrer (Nicht-) Bezahlung, später wurden auch Unterschiede zwischen Frauen, z. B. zwischen erwerbstätigen weißen Mittelschichtfrauen und migrantischen Frauen, die ihnen die Haus- und Care-Arbeit abnehmen, thematisiert (Lutz 2008). Darüber hinaus standen die körperliche und psychische Selbstbestimmung von Frauen (in Bezug auf Schwangerschaftsabbruch und Gewalt an Frauen) ebenso wie ökologische Fragen und die mangelnde Gleichberechtigung in Bezug auf die Teilhabe in allen gesellschaftlichen Bereichen im Zentrum feministisch-politischer Kämpfe. Der kollektive Austausch, auch in den selbstgegründeten autonomen Räumen, ermöglichte es, eigene und kollektive Erfahrungen zu deuten, Interessen und Ziele zu formulieren und gemeinsame und individuelle politische Aktivitäten zu legitimieren. Dabei waren, z. B. in der BRD, zu Beginn der Zweiten Frauenbewegung die Grenzen zwischen den verschiedenen sozialen Räumen fließend: „,[Das] Seminar und die Frauengruppe sind dasselbe“, erinnert sich Ulrike Prokop an die Anfänge feministischen Engagements an der Frankfurter Universität (Prokop 1986: 87, zit. n. Hark 2005: 225). Der wissenschaftliche Feminismus entwickelte sich dementsprechend zu einer Möglichkeit, das geteilte (Erfahrungs- und Alltags-)Wissen zu verknüpfen und herauszuarbeiten, inwiefern sich in den 
individuellen Erfahrungen Muster widerspiegeln, die auf gesellschaftliche Strukturen zurückzuführen und damit kollektiv kritisierbar sind.

An die Entstehungsnarration feministischer Theorie, die besagt, dass diese aus einer kollektiven Politisierung des Privaten und der Praxis hervorgegangen ist, kann in bestimmten feministischen Diskursen bis heute angeknüpft werden. Nur vor diesem Hintergrund sind Deutungskämpfe innerhalb feministischer Wissensproduktion - zwischen Praxis und Theorie, politischer Bewegung und Akademisierung, aber auch zwischen hegemonialen und marginalisierten Positionen - zu verstehen. Die in diese Kämpfe eingeschriebene epistemische Ungerechtigkeit, die auch die Verwendung von „Erfahrungswissen“ durchzieht und ordnet, wer sprechen und wissen darf und wer nicht, ist seit jeher zentraler Gegenstand feministischer Theorien und feministischen Aktivismus (Phipps 2016: 3). Den Positionen postkolonialer Feministinnen, die eine weitere Dezentrierung hegemonial gewordener weißer feministischer Diskurse fordern, gelingt es dabei allerdings nur selten, ins Zentrum feministischer Wissensproduktion vorzudringen (Gutiérrez Rodríguez 2000, Spivak 1988). Dies lässt sich verbinden mit Donna Haraways Konzept des „Situierten Wissens“ (1995 [1988]), das davon ausgeht, dass jede Art von Wissen und Erkenntnisanspruch einem bestimmten sozio-ökonomischen und politischen Kontext entspringt, sprich: durch diese situiert ist. Die Grenzziehungen zur Frage, welches Wissen dazu gehört und welches nicht, rühren von interessengeleiteten Machtstrategien her und beinhalten keine Annäherung an eine Wahrheit. Wenn Haraway sagt: „Geschichte ist eine Erzählung, die sich die Fans westlicher Kultur gegenseitig erzählen, Wissenschaft ist ein anfechtbarer Text und ein Machtfeld, der Inhalt ist die Form“ (Haraway 1995: 75), dann bringt sie ein zentrales feministisches Postulat auf den Punkt: Wissensansprüche und Wissenssubjekte sind historisch kontingent. An Stelle einer unmarkierten Wissensposition des Mannes und des Weißen, die Objektivität für sich beansprucht, plädiert sie für eine reflexive „Lehre verkörperter Objektivität [...], die paradoxen und kritisch-feministischen Wissenschaftsprojekten Raum böte“ (ebd.: 80). Feministische ,Objektivität" bedeutet dann, das eigene Wissen als situiertes aufzubrechen.

Ein zentrales Ziel feministischer Vorhaben war und ist es, grundlegende gesellschaftliche Veränderungen zu erkämpfen, Machtverhältnisse zu hinterfragen und zu transformieren, um alle Menschen gleichermaßen und gleichberechtigt an sozialen, politischen und ökonomischen Prozessen zu beteiligen und Diskriminierungen und Benachteiligungen auf verschiedenen Ebenen abzubauen. Gewalt, Ungerechtigkeiten und Diskriminierungen werden dabei sowohl mit Geschlecht in Verbindung gebracht als auch intersektional betrachtet: Sie sind untrennbar verknüpft mit anderen gesellschaftlichen Kategorien wie race, Klasse, Befähigung und Sexualität (Crenshaw 1991). Das bedeutet, Feminismus ist „transformative Politik“, wie es 1984 während eines Workshops beim 2. Internationalen und Interdisziplinären Frauenkongress in Groningen/Niederlande hieß (Bunch et al. 1985: 244, zit. n. Hark 2005: 20, FN 1). Zugleich ist Feminismus als „historisches Projekt“ (Hark 2005: 33) zu begreifen, da immer wieder begriffliche, theoretische und methodische Revisionen und Entwicklungen notwendig sind. Das heißt allerdings auch, dass die seit 
Jahrzehnten formulierten nicht-weißen, nicht-mittelschichtsbezogenen und trans/feministischen Positionen stärker als bisher überhaupt als feministische Positionen wahrgenommen und in feministische Diskurse eingebunden werden müssen. Nur dann kann das Projekt feministischer Wissensproduktion glaubwürdig für sich in Anspruch nehmen, die feministische Kritik des Feminismus konstitutiv zu benötigen.

\section{Akademischer Feminismus}

Seit den späten 1980er Jahren begannen einige Feministinnen für feministische Lehr- und Forschungsinhalte an Hochschulen der BRD zu kämpfen. Der sich entwickelnde akademische Feminismus basierte im Gegensatz zum wissenschaftlichen Feminismus nicht mehr auf der praktischen Auseinandersetzung der Akteur_innen in politischen Gruppen feministischer Bewegungen, sondern löste sich allmählich von ihr. Den Weg der Frauen- und Geschlechterforschung, später der Gender Studies, in die Universitäten der BRD begleitet dabei von Beginn an der hehre Anspruch, Praxis und Theorie weiter eng miteinander zu verknüpfen. Feminist_innen etablierten feministische Studieninhalte, zunächst in Form autonomer FrauenLesbenräume und -bibliotheken (Heimberg 2005), autonomer feministischer Seminare und autonomer feministischer Zeitschriften an der Schnittstelle von Praxis und Theorie[5], später folgten feministische Professuren, Gender-StudiesStudiengänge und interdisziplinäre Forschungszentren. Diese Entwicklung fällt zeitlich zusammen mit dem Schwächerwerden eines Teils der feministischen Bewegungen und der Übernahme einiger feministischer Forderungen in staatliche Gleichstellungspolitik in den 1990er Jahren (Dölling 2013: 33). Zeitgleich erstarken auch in der BRD queere und queer/feministische ebenso wie afrodeutsche und Schwarze Bewegungen, in denen Feministinnen eine wichtige Rolle spielten. Allerdings haben sich deren Themen und Positionen bis heute deutlich weniger in akademischen Diskursen verankern können als frühere feministische Foki.

Über die Jahrzehnte hat sich der akademische Feminismus von seinen Wurzeln im praxisbezogenen Feminismus weit entfernt. Die unterschiedlichen feministischen Wissensformen haben sich in die traditionelle Wissenshierarchie eingefügt, in der so etwas wie eine akademische ,reine Theorie höheres Ansehen erfährt als das abqualifizierte erfahrungsbasierte Wissen, das auch marginalisierte feministische Perspektiven umfasst. Akademische feministische Analysen blicken heute meist aus der Theorie auf gesellschaftliche Wirklichkeit. Im Gegensatz zu früher deutlicher artikulierten, radikal transformationsbezogenen feministischen Anliegen fehlt vielen heutigen Analysen in den Gender Studies außerdem aufgrund ihrer vorwiegenden Ausrichtung auf akademische Zusammenhänge und wegen ihres fehlenden Bewegungs- und Praxisbezugs der politisch radikalere Biss.[6] Die angeblich abnehmende Bedeutung feministischer Bewegungen (Dölling 2013) ist auch vor dem Hintergrund einer zunehmenden Neoliberalisierung der Gesellschaft zu verstehen, bei der das ökonomische Prinzip bis tief in die soziale Sphäre eindringt, wobei die immer stärker individualisierten Gesellschaftsmitglieder unter andauerndem Selbstoptimierungsdruck stehen, was u. a. auch kollektive Kämpfe erschwert. Hark (2005) benennt außerdem 
die Widersprüchlichkeit, ein radikal gesellschaftskritisches feministisches Projekt in die Strukturen einer Wissenschaftsinstitution einzufügen, die von Feminist_innen grundsätzlich als herrschaftsorientiert und patriarchal kritisiert werden. Befürchtet und bekämpft, aber häufig auch hingenommen, wird heute eine entpolitisierte Indienstnahme feministischen Wissens durch die Universitäten, worauf auch Dölling hinweist:

„Fragmentiert und eingebettet in neoliberale Diskurse ist das gesellschaftskritische Potenzial dieser feministischen Forderungen weitgehend verloren gegangen und diese können so - nicht zuletzt im akademisierten Feminismus, wie er zunehmend als Gender- und Diversity Studies an immer stärker betriebswirtschaftlich orientierten und organisierten Universitäten betrieben wird - als Teil postmoderner Regierungsweisen und Herrschaftsformen wirken."(Dölling 2013: 29f.)

Dies alles stellt die akademisierte feministische Wissensproduktion vor große Herausforderungen. [7] Diese feministische Position ist beispielhaft für hegemoniale feministische Debatten, indem sie die Geschlechterdimension in den Vordergrund rückt und die rassistische Verfasstheit der, indienstnehmenden Institution ausblendet (Kuria 2015, Ha et al. 2016, siehe auch das Gespräch „Rassismus, Klassenverhältnisse und Geschlecht an deutschen Hochschulen. Ein runder Tisch, der aneckt“ in diesem Heft). Dies berührt immer auch die Frage normativer Wissenschaft: Als politische Stimmen richten feministische Kritiken ihre grundlegende Gesellschaftskritik gegen patriarchale, rassistische, sexistische und homophobe kapitalistische Strukturen. Zugleich hat der transformationsbezogene politische Anspruch akademischer feministischer Wissensproduktion an Schärfe verloren. Nachdem die Institution Hochschule den engagierten Studierenden und Wissenschaftler_innen nach harten und erbitterten Kämpfen zugestanden hatte, in einigen Disziplinen Frauen- und Geschlechterforschung zu etablieren, scheint nun ihre Zugehörigkeit zu einer Hochschule die feministische Wissensproduktion zu verändern, wobei die traditionelle Hierarchisierung von Wissensformen übernommen wird. Es wird deutlich, dass es ein Irrtum war, anzunehmen, dass feministische Kritik ,unschuldig' wäre: Sara Ahmed weist darauf hin, dass Kritik zur Performance verkümmert, wenn jemand sie sich als Eigenschaft und Eigentum zuschreibt (Ahmed 2004, Abs. 10). Kritik muss sich im Tun zeigen. Zu fragen ist also, wie sich feministische Wissenschaft auch innerhalb der Akademie als kritische Stimme weiterentwickeln und dabei ihren gesellschaftskritischen Anspruch aufrechterhalten kann. Nicht zuletzt die Etablierung der Gender Studies, die als gefälliger und mainstream-tauglicher gelten als die frühere feministische Geschlechterforschung, aber auch inhaltliche Brüche wie die konstruktivistische und poststrukturalistische Wende seit den frühen 1990er Jahren[8] werden dafür verantwortlich gemacht, dass Feminismus heute in der öffentlichen Wahrnehmung entweder als eher harmlos und nicht mehr radikal erscheint, oder in neu erstarkenden, antifeministischen Angriffen als überflüssig, ideologisch überfrachtet und veraltet dargestellt wird (Hark/Villa 2015). In diesen Annahmen spiegeln sich aber auch feministische Grabenkämpfe um den angeblichen Verlust ,des' feministischen Subjekts ,Frau' wider, verbunden sowohl mit rassismusblinden Annahmen als auch mit Transphobie und Unreflektiertheit in Bezug auf Heteronormativität. 


\section{Neue Horizonte feministischer Wissensproduktion}

Ein spannendes Beispiel für einen neuerlichen Versuch, Theorie bewegungsbeziehungsweise praxisnah weiterzuentwickeln, ist die aktuelle Bewegung der „Precarias a la deriva“ (2014), die sich 2002 als loser Zusammenschluss im Zuge des spanischen Generalstreiks in Madrid gegründet haben. In ihrer „militanten Untersuchung“ (ebd.: 35) von Prekarisierung und prekarisierter, meist von Frauen erledigter Fürsorgearbeit, die sie in sehr umfassendem Sinne verstehen, stellen sie fest:

„Aktuell besteht eine der fundamentalen biopolitischen Herausforderungen darin, eine Kritik der gegenwärtigen Organisation von Geschlecht, Aufmerksamkeit und Fürsorge zu formulieren. Zu entwickeln ist ebenso eine Praxis, die von diesen als Zusammenhang ausgeht und sie rekombiniert, um neue, freiere und kooperative Formen des Affekts zu produzieren, in deren Zentrum die Fürsorge steht, ohne sie aber von Geschlecht und Kommunikation zu separieren." (Precarias a la deriva 2005: 7)

Fürsorge betrachten sie als Grundlage aller menschlichen Existenz und aller sozialen Beziehungen: als „das, was das Leben möglich macht“ (ebd.). Sie schlagen vor, innerhalb der Organisierung des Sozialen die Menschen an erste Stelle zu setzen und dabei dafür zu sorgen, dass alle primären Bedürfnisse, vom Zugang zu Nahrung und Versorgung bis zum „Recht auf Affekte“, nachhaltig befriedigt werden (ebd.: 8). In der Gesellschaft sollten die Entfaltung als menschliche Wesen und die Orientierung an den menschlichen Wünschen und Bedürfnissen oberste Priorität haben, was den Zugang zu Wissen und Bildung ebenso einschließt wie die weltweite Bewegungsfreiheit. Um auf die zahlreichen, oft unsichtbaren Fürsorgetätigkeiten von Frauen aufmerksam zu machen, die bisher kaum gesellschaftliche Wertschätzung und Anerkennung erhalten, und alle Praktiken, die als Fürsorgearbeit zählen können, auch als solche zu denken, schlagen sie einen „Fürsorge-Streik“ vor. Die Praxis des Streiks soll dazu genutzt werden, kollektiv Alternativen zu entwickeln und so darauf aufmerksam zu machen, dass „Fürsorge kein häusliches Problem [ist], sondern eine öffentliche Angelegenheit und ein Generator für Auseinandersetzungen“ (ebd.: 9). Indem sie in ihren als „dérive“ verstandenen und praktizierten Suchbewegungen bei den individuellen Lebensbedingungen und Lebensformen ansetzen, um „die Existenz zu politisieren" (Precarias a la deriva 2014: $38 \mathrm{f}$.), beziehen sie alte feministische Herangehensweisen einer Politisierung scheinbar individueller Angelegenheiten auf heutige Zeiten extremer Vereinzelung und Prekarisierung der Lebensbedingungen. Ihre schriftlichen und audiovisuellen Aufzeichnungen der subjektiven Perspektiven sind zugleich Interventionen und Produktionen eines teilbaren Wissens, das auf neue Weise zirkulieren und zu Politisierung und neuen Interventionen führen kann (Trott 2013: 123).

Projekte wie dieses verdeutlichen, wie feministische Praxis auch heute feministische Wissensproduktion vorantreibt. Insbesondere die Debatte um Fürsorgearbeit (care) an der Schnittstelle vom praxisbezogenen zum akademischen Feminismus erscheint zurzeit besonders produktiv (vgl. z. B. das caGE-Projekt[9]). Die Precarias a la deriva geben eine von vielen 
Antworten auf die Frage, wie sich antirassistische, queer/feministische politische Bewegungen wieder stärker „auf der Schwelle [zur Universität] [...] platzieren“ (Hark 2005: 245) können, wie es in den frühen Versuchen des akademisch werdenden Feminismus hieß.

Im Weg steht einem solchen Anspruch aktuell die Institutionalisierung und hegemoniale Position des akademischen Feminismus und ausgewählter Wissensformen, die ganz ohne Bewegungs- und Praxisbezug kanonisiert studiert und forschend weiter entwickelt werden können. Diese Ablösung feministischer Wissensproduktion von Erfahrungsbezügen erzeugt häufig ein gewissermaßen entkörpertes, objektiviertes und kaum noch transformationsbezogenes Wissen, das die eigene Herrschaftsposition und die Verstrickung akademischer feministischer Wissensproduktion in bestehende Strukturen nicht reflektiert und dadurch reaffirmiert.

Feministische Wissensproduktion könnte ihrem reflexiven Anspruch wieder mehr gerecht werden und zu neuer politischer Schärfe gelangen, wenn feministische Theorie und die unterschiedlichen feministischen Bewegungen den wechselseitigen Austauschprozess auf Augenhöhe wieder stärker mit Leben erfüllen würden - denn Kritik ist etwas, was getan werden muss (Ahmed 2004). Dies würde feministischen Akademiker_innen abverlangen, vom hohen akademischen Ross herabzusteigen und die eigene gesellschaftliche Position wieder stärker in die Analysen einzubeziehen, aber auch, die Sensoren auszufahren für die zahlreichen gesellschaftspolitischen Bewegungen, die wieder stärker in die feministische Wissensproduktion einfließen müssten. Akteur_innen politischer queer/feministischer und antirassistischer Bewegungen sollten sich wieder stärker selbst dazu ermächtigen, die feministische Wissensproduktion selbstbewusst mitzugestalten und sie sich nicht länger aus den Händen nehmen zu lassen.

\section{Endnoten}

[1] Jan Simon Hutta und Johanna Hoerning danke ich für Anregungen und Hinweise, die ich längst nicht alle berücksichtigen konnte. Ich freue mich, wenn sich unsere Diskussionen dazu in der Zukunft fortsetzen.

[2] Auch das Wissen über Marginalisierungserfahrungen und dessen Verwendung in (wissenschaftlichen) Diskursen trägt nicht automatisch dazu bei, machtvolle Dominanzverhältnisse und soziale Schräglagen zu verändern. Es kann im Gegenteil sogar dazu genutzt werden, konservative Politiken voranzutreiben. So werden beispielsweise Opfererzählungen von Prostituierten in Politiken für restriktivere Prostitutionsgesetze genutzt. Vom selektiven Anführen subjektiver Erfahrungen anderer profitieren dann die Sprecher_innen, die sich dabei nicht selten in Opposition zu den Interessen der Marginalisierten stellen, die von Graswurzelorganisationen artikuliert werden (Phipps 2016: 6; 8).

[3] Vgl. das Doppelschwerpunktheft zu Trans/Feminisms der Zeitschrift Transgender Studies Quarterly 3: 1-2 (2016). Zum Ausschluss von Trans* aus feministischen Räumen vgl. Phipps 2016: $8 \mathrm{ff}$., Schuster 2010: $278 \mathrm{ff}$. Ein konstruktives Beispiel für trans*inklusive Frauen*-Raumpolitiken findet sich in der GLADT-Broschüre Frauenräume und die Diskussion um Trans*-Offenheit (2011).

[4] Hier und im Folgenden verwende ich das Konzept „Frau(en)“ in seiner vielfältigsten Bedeutung, was die Reflexion über dessen soziale Konstruiertheit und Unabgeschlossenheit beinhaltet. Gemeint sind damit also ausdrücklich alle Personen, die sich selbst als „Frauen“ bezeichnen - auch wenn ihre Teilhabe am feministischen Projekt stets umkämpft war und bis heute ist. 
[5] Z. B. der an.schläge in Wien (seit 1986 bis heute), der Krampfader in Kassel (seit 1988 bis heute), der Ihrsinn (1989 - 2004), der Xanthippe in Marburg (1991 - 98).

[6] Dies lässt sich auch am Beispiel der deutschsprachigen Rezeption der Queer Theory verdeutlichen. Im Gegensatz zur Entwicklung feministischer Wissensproduktion sind die Wissensproduktionen queerer Bewegungen und Queer Studies, die aus anglo-amerikanischen Bewegungskontexten und akademischen Auseinandersetzungen hervorgegangen sind, seit den späten 1990er Jahren im deutschsprachigen Raum zunächst als akademische Diskurse rezipiert worden, anfangs z. B. durch die Rezeption der Übersetzungen von Adrienne Rich (1980, deutsch: 1993) und Judith Butler (1991; 1997) sowie der Übersetzungen von Rubin, de Lauretis, Butler, Sedgwick, Halperin, Dinshaw, Bravmann, Traub, Creech in Kraß (Hg.) (2003). Diese Texte wurden und werden heute in einigen Bewegungskontexten wiederum als wichtiges theoretisches Hintergrundwissen durchgearbeitet. Hier fehlte in der deutschsprachigen Rezeption meist von Beginn an die Rückkopplung aus den Bewegungen in die akademischen Diskurse hinein. Des Weiteren spielt eine Rolle, dass die Formierung queerer Bewegungen in den verschiedenen nationalen Kontexten auf ganz unterschiedliche Weise verlief und verläuft. Diese Rezeptionsgeschichte könnte einer der Gründe dafür sein, dass es bis heute an deutschsprachigen Universitäten - außer in Verknüpfung mit den Gender Studies - noch nicht gelungen ist, queer/feministische Studien eigenständig institutionell zu verankern, z. B. in Form eigener Studiengänge und unbefristeter Dozent_innenstellen und Professuren.

[7] Einige Positionen dazu werden z. B. in den feministischen studien 31/1 (2013) diskutiert.

[8] Vgl. Butler (1991); feministische studien 11/2 (1993) für den deutschsprachigen Kontext und Benhabib et al. (1993) für die angloamerikanische Diskussion.

[9] „Care, Gender and Green Economy. Forschungsperspektiven und Chancengerechtigkeit nachhaltigen Wirtschaftens“ (CaGE), gefördert durch das BMBF, 2013-14: www.cageonline.de.

\section{Autor innen}

Nina Schuster ist Soziologin und forscht an der Schnittstelle von stadtsoziologischen und queer/feministischen Theorien zur sozialen und räumlich-materiellen Produktion sozialer Ungleichheit.

nina.schuster@tu-dortmund.de

\section{Literatur}

Ahmed, Sara (2004): Declarations of whiteness: The non-performativity of anti-racism. In: Borderlands 3/2. http://www.borderlands.net.au/vol3no2_2004/ahmed_declarations. htm (letzter Zugriff am 23.08.2016).

Benhabib, Seyla et al. (1994): Der Streit um Differenz. Feminismus und Postmoderne in der Gegenwart. Frankfurt/Main: Fischer.

Bunch, Charlotte/Carillo, Roxana/Guinée, Ied (1985): Feminist perspectives. Report on the feminist perspectives working group on the closing plenary. In: Women's Studies International Forum 8/4, 243-247.

Butler, Judith (1991 [1990]): Das Unbehagen der Geschlechter. Frankfurt/Main: Suhrkamp. (Übers. Kathrina Menke)

Butler, Judith (1997 [1993]): Körper von Gewicht. Die diskursiven Grenzen des Geschlechts. Frankfurt am Main: Suhrkamp. (Übers. Karin Wördemann)

Crenshaw, Kimberlé Williams (1991): Mapping the margins: Intersectionality, identity politics, and violence against Women of Color. In: Stanford Law Review 43/6, 1241-1299.

Dölling, Irene (2013): 30 Jahre feministische Studien: Wie mit dem feministischen Erbe umgehen? In: feministische studien 31/1, 29-34.

Freire, Paolo (1971 [1970]): Pädagogik der Unterdrückten. Stuttgart: Kreuz-Verlag.

GLADT (2011): Frauenräume und die Diskussion um Trans*-Offenheit. http://www.gladt. de/seite/268126/trans-in-frauenr\%C3\%A4umen.html (letzter Zugriff am 7.10.2016). 
Gutiérrez Rodríguez, Encarnación (2000): Fallstricke des Feminismus. Das Denken „kritischer Differenzen“ ohne geopolitische Kontextualisierung. Einige Überlegungen zur Rezeption antirassistischer und postkolonialer Kritik. In: polylog. Forum für interkulturelle Philosophie 2. http://them.polylog.org/2/age-de.htm (letzter Zugriff am 7.10.2016).

Ha, Kien Nghi / Ha, Noa / Mesghena, Mekonnen (Hg.) (2016): Dossier „Geschlossene Gesellschaft? Exklusion und rassistische Diskriminierung an deutschen Universitäten.“ Berlin: Heinrich-Böll-Stiftung.

Haraway, Donna (1995 [1988]): Situiertes Wissen. Die Wissenschaftsfrage im Feminismus und das Privileg einer partialen Perspektive (Übers. Helga Kelle). In: Donna Haraway, Die Neuerfindung der Natur. Primaten, Cyborgs und Frauen (hg. und eingeleitet von Carmen Hammer und Immanuel Stiess). Frankfurt am Main und New York: Campus, 73-97.

Hark, Sabine (2005): Dissidente Partizipation. Eine Diskursgeschichte des Feminismus. Frankfurt am Main: Suhrkamp.

Hark, Sabine / Villa, Paula-Irene (2015): Eine Frage an und für unsere Zeit. Verstörende Gender Studies und symptomatische Missverständnisse. In: Sabine Hark / Paula-Irene Villa (Hg.), Anti-Genderismus Sexualität und Geschlecht als Schauplätze aktueller politischer Auseinandersetzungen. Bielefeld: transcript, 15-39.

Heimberg, Anke (Hg.) (2005): „...das erste und einzige feministische Archiv in Marburg“. 15 Jahre Feministisches Archiv Marburg. Ein Projekt der Studentinnen- und Frauenbewegung. Marburg: BdWi-Verlag.

Kindon, Sara Louise / Pain, Rachel / Kesby, Mike (2009): Participatory Action Research. In: Rob Kitchen / Nigel Thrift (Hg.), International Encyclopedia of Human Geography. Amsterdam u. a.: Elsevier, 90-95.

Kraß, Andreas (Hg.) (2003): Queer Denken. Gegen die Ordnung der Sexualität. Frankfurt am Main: Suhrkamp.

Kuria, Emily Ngubia (2015): eingeschrieben. Zeichen setzen gegen Rassismus an deutschen Hochschulen. Berlin: w_orten \& meer.

Lutz, Helma (2008): Vom Weltmarkt in den Privathaushalt. Die neuen Dienstmädchen im Zeitalter der Globalisierung. Opladen u. a.: Barbara Budrich.

Phipps, Alison (2016): Whose personal is more political? Experience in contemporary feminist politics. In: Feminist Theory. DOI: 10.1177/1464700116663831.

Precarias a la deriva (2005): Ein sehr vorsichtiger Streik um sehr viel Fürsorge. Vier Hypothesen (Übers. Jens Kastner). www.republicart.net/disc/precariat/precariaso2_ de.htm (letzter Zugriff am 12.07.2016)

Precarias a la deriva (2014): Was ist dein Streik? Militante Streifzüge durch die Kreisläufe der Prekarität. Wien: transversal texts.

Rich, Adrienne (1980): Compulsory heterosexuality and lesbian existence. In: Signs: Journal of Women in Culture and Society 5, 631-66o.

Schuster, Nina (2010): Andere Räume. Soziale Praktiken der Raumproduktion von Drag Kings und Transgender. Bielefeld: transcript.

Spivak, Gayatri (1988): Can the subaltern speak? In: Cary Nelson / Lawrence Grossberg (Hg.), Marxism and the Interpretation of Culture. London: Macmillan, 271-313.

Stryker, Susan / Bettcher, Talia M. (2016). Introduction. Trans/Feminism. In: TSQ Transgender Studies Quarterly 3/1-2, 5-14.

Trott, Ben (2013): Gefühle, Affekte, Feminisierung: Zeitgenössische Interventionen in die geschlechtliche Arbeitsteilung. In: Beate Binder et al. (Hg.), Eingreifen, Kritisieren, Verändern!? Interventionen ethnographisch und gendertheoretisch. Münster: Westfälisches Dampfboot, 104-115. 
